\title{
On the Durability of Sealable Circular Concrete Structures under Chloride Environment
}

\author{
Changwen Mi, ${ }^{1}$ Kaikai Shao, ${ }^{1}$ and Xiaobao $\mathrm{Li}^{2}$ \\ ${ }^{1}$ Jiangsu Key Laboratory of Engineering Mechanics, Department of Engineering Mechanics, Southeast University, \\ Nanjing, Jiangsu 210096, China \\ ${ }^{2}$ Department of Mechanical Engineering, University of Houston, Houston, TX 77204, USA
}

Correspondence should be addressed to Changwen Mi; mi@seu.edu.cn

Received 3 March 2015; Accepted 26 May 2015

Academic Editor: Ana S. Guimarães

Copyright (C) 2015 Changwen Mi et al. This is an open access article distributed under the Creative Commons Attribution License, which permits unrestricted use, distribution, and reproduction in any medium, provided the original work is properly cited.

Reinforcement corrosion resulting from chloride attack is one major mechanism that compromises concrete durability. Numerical methods were commonly used for tackling Fick's diffusion equations. In this paper, we developed a Crank-Nicolson based finite difference scheme suitable for circular concrete structures. Both a time-dependent surface chloride model and diffusivity were considered. The impact of an ideal sealer on chloride redistribution was further investigated. Results suggested that the chloride threshold depth in a concrete structure is greatly affected by the radius of curvature, environment severity, and diffusivity. For sealable concrete structures, both the sealer application timing and location are of great importance.

\section{Introduction}

The reinforcement steel in concrete structures is protected from adverse environments by a thin layer of passive oxide film. Exposure to aggressive species, for example, chloride anions, may result in the breakdown of the naturally formed oxide $[1,2]$. The volume expansion due to corrosion products causes an excessive stress state. In severe cases, cracking and even spalling of the concrete cover occur. The durability of a concrete structure is thus compromised due to the possible strength degradation of rusted reinforcements [3].

A threshold level of chlorides (CTL) is one of the necessary conditions for reinforcement corrosion [4]. The specific values defined for the CTL, however, vary from one another in the literature. For example, a threshold value of $0.594 \mathrm{~kg} / \mathrm{m}^{3}$ was used by Kassir and Ghosn [5] and Phurkhao and Kassir [6]. Moriwake [7] defined a larger value of $2.0 \mathrm{~kg} / \mathrm{m}^{3}$ for crack initiation due to steel corrosion. Song et al. [3] used both values as well as an intermediate CTL of $1.2 \mathrm{~kg} / \mathrm{m}^{3}$ for predicting the service life of repaired concrete structures. As Alonso et al. [8] summarized, the CTL of a concrete structure is affected by many factors such as the oxide condition of reinforcements, concrete quality, and environmental severity.
Chloride ingress in concretes is primarily associated with two mechanisms: diffusion [9] and hydraulic solution diffusivity [10]. Concentration gradient and capillary suction would be the driving force of these two mechanisms in absence of pressure in unsaturated concrete. Under pressure phenomena would be even more complex. For each mechanism, a certain extent of chloride binding may occur, depending on the microstructural porosity and the moisture state of a concrete structure [11, 12]. Nonetheless, quantitative models are still being developed $[13,14]$ and the detailed mechanism of chloride binding on the durability of concrete structures remains unclarified [4].

Without considering the effect of chloride binding, chloride diffusion in concrete is relatively a well-understood mechanism, benefitted from Fick's fundamental diffusion laws [15]. A large amount of literature studies was devoted to this line of research. Analytical solutions have been developed for simple geometric domains, boundary conditions, initial chloride distribution, and diffusivity $[5,6,15]$. They are often used as benchmark examples for calibrating the reliability of more complicated solutions.

A large portion of the relevant literature studies is concerned with one-dimensional diffusion problems $[3,14,16$, 
17]. More recently, considerable efforts have also been made to numerically resolve two-dimensional problems of different domains. The numerical methods that were employed include finite difference method [18], finite element method [13, 19, 20], finite point (meshless) method [21], cellular automata [22], and boundary element method [23]. Analytical solutions were also being under development for cases with an apparent diffusion constant [24, 25].

Less effort has been directed towards the study of circular columns, even though they form a very common type of structural elements in civil infrastructures, as opposed to rectangular and elliptic ones. The chloride diffusion inside a two-dimensional circular domain remains elusive. This is the goal of the present study.

When compared to analytical solutions, one distinct advantage of a numerical method is its capability to accommodate complex diffusivity model, surface chloride model, and initial chloride distribution. Experimental data from field studies has revealed that both the diffusivity [26] and surface chloride concentration [27] vary with concrete age. In the present work, we borrowed the time-dependent diffusivity of Song et al. [3], which was slightly adapted from Bentz and Thomas [28].

For surface chloride evolution, the situation is more complicated. Environmental factors that affect the evolutionary history of the surface chloride include geographic location of a concrete structure, accessibility to rinsing precipitations, relative location inside an infrastructure, season, and wetting and drying cycles. A few regression models, including exponential [5], ramp-type [6], logarithmic [3, 29], and power functions [30], have been proposed in the literature by fitting to experimental measurements in the least squares fashion. While each of these surface chloride models can be incorporated into the present study, we specifically focused on the ramp-type [6] and log-type [31]. They represent a deicing salt induced chloride and marine environment, respectively.

Among all available solution strategies, the finite difference method is the simplest one in terms of the easiness in mathematical implementation $[3,23,32]$. In this work, we developed in detail a Crank-Nicolson based finite difference scheme to numerically solve the axially symmetric diffusion equation defined in a circular domain. The CTL value of $1.2 \mathrm{~kg} / \mathrm{m}^{3}$ [3] was used. In addition to the chloride diffusion in an original unpolluted concrete, numerical experiments were also performed for the chloride redistribution following a sealer application. The sealer was applied at either the outer surface or at a certain depth.

Simulation results indicated that the chloride distribution strongly depends on concrete size, surface chloride evolution, diffusivity, initial chloride distribution, and sealer application timing and location. The most effective means for slowing down chloride diffusion is to elevate concrete quality. To prevent chlorides from further penetrating, a sealer application is the best option. In this case, the sealer should be applied early and as close to the outermost reinforcements as possible.

The remainder of this paper is structured as follows. In Section 2 the numerical scheme that we proposed for the analysis of chloride diffusion in circular concrete structures is presented in detail. In Section 3 a variety of numerical experiments detailing the chloride diffusion in both unsealed and sealed concretes are reported and discussed and finally, in Section 4, conclusions are provided and future works described.

\section{Method of Solution}

2.1. Crank-Nicolson Scheme for Chloride Diffusion in a Circular Domain. According to Fick's first law [15], the concentration gradient of chlorides serves as the driving force to their spatial diffusion in concrete structures:

$$
\mathbf{J}(\mathbf{x}, t)=-D(t) \nabla C(\mathbf{x}, t),
$$

where $\mathbf{J}$ is the diffusion flux of chloride anions, $C$ the chloride concentration, and $D$ the diffusivity. Generally, both $\mathbf{J}$ and $C$ are functions of space and time whereas $D$ is typically treated as a time-dependent variable only.

In the absence of chloride binding the conservation of mass inside a differential volume dictates that the rate of change of chlorides must be balanced by the divergence of the diffusion flux. The resultant partial differential equation is referred to as Fick's second law of diffusion:

$$
\frac{\partial C(\mathbf{x}, t)}{\partial t}=D(t) \nabla^{2} C(\mathbf{x}, t)
$$

In this work, we focused on circular and homogeneous concretes. From the practical point of view, it seems reasonable to assume that the chloride diffusion process is also symmetric about the centroidal axis. Under these assumptions, (2) can be rewritten as

$$
\frac{\partial C(r, t)}{\partial t}=D(t)\left(\frac{\partial^{2} C(r, t)}{\partial r^{2}}+\frac{1}{r} \frac{\partial C(r, t)}{\partial r}\right) .
$$

Unlike the one-dimensional diffusion problem, the analytical solution to (3) is unavailable in the literature. Numerical alternatives are employed instead. For the reasons stated in Section 1, we decided to employ the finite difference method and aimed to develop a numerical model on the basis of Crank-Nicolson scheme [32, 33].

We divided the radius $R$ of a circular domain into $M$ segments, each with the same length. As a result, there are a total number of $(M+1)$ spatial nodes. The central idea of the Crank-Nicolson scheme is to consider the diffusion equation (3) at an arbitrary nonboundary node and halfway between two adjacent temporal steps:

$$
\frac{\partial C_{i, j-1 / 2}}{\partial t}=D_{j-1 / 2}\left(\frac{\partial^{2} C_{i, j-1 / 2}}{\partial r^{2}}+\frac{1}{(i-1) h} \frac{\partial C_{i, j-1 / 2}}{\partial r}\right),
$$

where the subscripts $i(=1,2, \ldots, M+1)$ and $j(=1,2, \ldots)$ denote the spatial and temporal indices, respectively. They are related to the real coordinates through $r=(i-1) h$ and $t=(j-1) \tau$, where $h$ and $\tau$ are the spatial and temporal step increments. The temporal derivative is approximated by the centered difference scheme:

$$
\frac{\partial C_{i, j-1 / 2}}{\partial t}=\frac{C_{i, j}-C_{i, j-1}}{\tau} .
$$


The first- and second-order spatial derivatives in (4) are approximated by temporally averaging a one-sided and centered difference scheme over two contiguous temporal steps, respectively,

$$
\begin{aligned}
& \frac{\partial C_{i, j-1 / 2}}{\partial r}=\frac{1}{2}\left(\frac{C_{i+1, j-1}-C_{i, j-1}}{h}+\frac{C_{i+1, j}-C_{i, j}}{h}\right), \\
& \frac{\partial^{2} C_{i, j-1 / 2}}{\partial r^{2}}=\frac{1}{2}\left(\frac{C_{i-1, j-1}-2 C_{i, j-1}+C_{i+1, j-1}}{h^{2}}\right. \\
& \left.+\frac{C_{i-1, j}-2 C_{i, j}+C_{i+1, j}}{h^{2}}\right) .
\end{aligned}
$$

Substituting (5) and (6) back into (4) and solving for the concentrations at the $j$ th temporal step, we have

$$
\begin{aligned}
& a_{i} C_{i-1, j}+b_{i} C_{i, j}+c_{i} C_{i+1, j} \\
& \quad=-a_{i} C_{i-1, j-1}+\left(2-b_{i}\right) C_{i, j-1}-c_{i} C_{i+1, j-1},
\end{aligned}
$$

where $a_{i}, b_{i}$, and $c_{i}$ are functions of the diffusivity $\left(D_{j-1 / 2}\right)$, the Courant number $\left(\tau / h^{2}\right)$, and the spatial node index $(i=$ $2,3, \ldots, M)$. For brevity, they are tabulated in Appendix A.

This numerical scheme is implicit since at an arbitrary temporal step the concentrations at three neighboring nodes are always coupled. Thus, care should be practiced for the boundary nodes. The chloride concentration at the outer boundary is governed by the temporal evolution of surface chlorides:

$$
C_{M+1, j}=C_{s}((j-1) \tau)
$$

where $C_{s}(t)$ represents a time-dependent surface concentration model. As a result, the $M t h$ equation of (7) must be reformulated to reflect this condition:

$$
\begin{aligned}
a_{M} C_{M-1, j}+b_{M} C_{M, j}= & -a_{M} C_{M-1, j-1} \\
& +\left(2-b_{M}\right) C_{M, j-1} \\
& -c_{M}\left(C_{M+1, j-1}+C_{M+1, j}\right) .
\end{aligned}
$$

Given the symmetry property of the present problem, the concentration gradient vanishes at $r=0$. In mathematical context, this represents a Neumann boundary condition [32]:

$$
\frac{\partial C(0, t)}{\partial r}=0
$$

At $r=0(i=1)$, we may start from a centered approximation for both spatial derivatives

$$
\begin{aligned}
& \frac{\partial C_{1, j-1 / 2}}{\partial r}=\frac{1}{2}\left(\frac{C_{2, j-1}-C_{0, j-1}}{2 h}+\frac{C_{2, j}-C_{0, j}}{2 h}\right)=0, \\
& \frac{\partial^{2} C_{1, j-1 / 2}}{\partial r^{2}}=\frac{1}{2}\left(\frac{C_{0, j-1}-2 C_{1, j-1}+C_{2, j-1}}{h^{2}}\right. \\
& \left.\quad+\frac{C_{0, j}-2 C_{1, j}+C_{2, j}}{h^{2}}\right) .
\end{aligned}
$$

The equality to zero in the first-order derivative is obviously a result of the Neumann boundary condition (10). To proceed, those concentrations that correspond to the undefined node $(i=0)$ need to be eliminated. Multiplying the first equation by $2 / h$ and adding to the second one, we managed to derive a new finite difference approximation for the second-order spatial derivative at $r=0$ :

$$
\frac{\partial^{2} C_{1, j-1 / 2}}{\partial r^{2}}=\frac{C_{2, j-1}-C_{1, j-1}}{h^{2}}+\frac{C_{2, j}-C_{1, j}}{h^{2}}
$$

Now consider the original diffusion equation (3) for $r=$ 0 . The term involving the first-order spatial derivative is addressed by limit analysis. Upon application of L'Hôpital's rule for $r \rightarrow 0$, this term's limit turns out to be another second-order spatial derivative. Replacing in (3) the temporal derivative with (5) and the second-order spatial derivatives with (12), an evolutionary scheme for $r=0$ can be developed:

$$
\begin{aligned}
b_{1} C_{1, j}+c_{1} C_{2, j}= & \left(\frac{1}{2}-\frac{\tau}{h^{2}} D_{j-1 / 2}\right) C_{1, j-1} \\
& +\frac{\tau}{h^{2}} D_{j-1 / 2} C_{2, j-1},
\end{aligned}
$$

where $b_{1}$ and $c_{1}$ are functions of the diffusivity and the Courant number. Both are tabulated in Appendix A.

Up to the present, we constructed $M$ linear equations regarding the chloride concentration at an arbitrary temporal step, that is, $(M-2)$ equations from (7), equations (9) and (13). The total number of unknowns is the same; that is, $C_{1, j} \sim$ $C_{M, j}$. Recall that the chloride concentration at the largest node number $(M+1)$ is dictated by the surface chloride evolution. To facilitate the solution procedure, this system of linear equations was reformulated into conventional matrix form. The reader is kindly invited to refer to Appendix A for further details.

2.2. Sealer Application. In engineering practice, sealers, coating, and membranes are often employed as means of maintenance. The idea is to prevent or at least to slow down chloride ingress toward reinforcement steels inside a concrete structure. In physical context, the prevention of chloride transport denotes that the diffusion flux is zero across a sealing interface. In view of (1), a sealer application annihilates the concentration gradient of chloride ions in position. Such a condition is mathematically identical to Neumann boundary condition that was applied at the center of a circular concrete.

Therefore, the approximation schemes used for the concrete center $r=0$, that is, (11) through (13), can be adapted for a sealing interface. If a sealer is applied at the outer surface of the concrete, only a single spatial interval needs to be considered. Otherwise, two separate regions must be taken into account. As a result, the numerical scheme designed for an original concrete structure must be revised to reflect Neumann boundary condition associated with the sealing interface. For conciseness, the revised scheme is outlined in Appendix B. 
2.3. Time-Dependent Surface Chloride Evolution. To implement the numerical scheme developed in the previous section, we first need to quantify an evolution model for the surface chloride concentration, an initial chloride distribution, and a time-dependent diffusivity model. As briefly discussed in Section 1, several regression models have been proposed in the literature to simulate the temporal evolution of surface chloride ions $[3,5,6,29,30]$. Although any of them can be implemented, we specifically focused on two surface chloride models. The first is a ramp-type surface concentration model [6]:

$$
\begin{aligned}
& C_{s}(t)=C_{0} \frac{t}{t_{0}}, \quad t \leq t_{0}, \\
& C_{s}(t)=C_{0}, \quad t>t_{0} .
\end{aligned}
$$

This model predicts that surface concentration increases linearly as exposure time for the first $\left(t_{0}=5\right)$ years of a fresh concrete structure, beyond which the surface concentration remains saturated $\left(C_{0}=5.343 \mathrm{~kg} / \mathrm{m}^{3}\right)$. The two parameters were extracted by fitting the proposed function to the measured surface chloride data from 15 bridge decks in the snow belt region of the United States [27]. The source of chloride ions is due primarily to deicing salts applied on the bridges during snow seasons. Given the initially increasing and subsequently fluctuating nature of experimental surface chloride measurements [27], (14) seems to represent an improved regression over its exponential counterpart [5].

For comparison purpose, the logarithmic model designed for concrete structures under marine environment was also employed $[3,31]$ :

$$
C_{s}(t)=\alpha \ln (\beta t+1),
$$

where $\alpha$ and $\beta$ are fitting parameters that are functions of the distance from seawater. For the case of zero distance, $\alpha=$ $1.52 \mathrm{~kg} / \mathrm{m}^{3}$ and $\beta=3.77$ year $^{-1}$. The surface concentration predicted by (15) monotonically increases as exposure time but at a decreasing rate, for example, $\mathrm{d}_{s} / \mathrm{d} t \sim 1 / t$. Such a model is appropriate for concrete structures subjected to wetting and drying cycles.

2.4. Initial Chloride Distribution and Time-Dependent Diffusivity. For concretes free of sealer applications, an initially chloride-free condition was always assumed. The chloride distribution immediately prior to a sealer application action was undoubtedly treated as the initial condition of the subsequent chloride evolution.

As evidenced by Fick's laws (1) and (2), the evolution of chloride concentration interior of a concrete structure is governed by the time-dependent diffusivity. Mathematically, diffusivity is the linear proportionality between diffusion flux and concentration gradient. In physical terms, it means how difficult or how easy chlorides can spatially redistribute. It is proposed by Bentz and Thomas [28] that chloride diffusivity decays as a power function for the first 25 years and stays constant afterwards. Such a dependent pattern is believed to be a result of the void growth and coalescence in a concrete structure due to cement hydration [31].
In this work, a slightly modified version [3] of the timedependent diffusivity [28] is used:

$$
\begin{aligned}
& D_{w / c}(t)=D_{w / c}\left(\frac{t_{R}}{t}\right)^{0.2}, \quad t \leq 30 \text { years } \\
& D_{w / c}(t)=D_{w / c}\left(\frac{t_{R}}{30}\right)^{0.2}, \quad t>30 \text { years }
\end{aligned}
$$

where $D_{w / c}$ is the reference diffusivity when $t=t_{R}=28 / 365$ years and a function of the water to cementitious material ratio $w / c[3]$ :

$$
D_{w / c}=10^{(-12.06+2.4 w / c)} .
$$

Typical range of $w / c$ is between 0.3 and 0.5 . In the absence of fly ash and slag, the decaying rate of diffusivity was assumed as 0.2 [3].

\section{Results and Discussion}

Based on the finite difference scheme developed in the previous section, we performed a variety of numerical experiments for investigating the influential factors on chloride diffusion in circular concrete structures. These factors include curvature of radius, water to cement ratio, surface concentration model, and sealer application timing and location.

To proceed, numerical examples were first implemented to examine the reliability of the proposed numerical scheme with respect to both spatial and temporal step sizes. Figure 1 reports chloride concentration as a function of spatial step for two distinct temporal steps. Concentrations were all sampled at the perimeter $0.1 \mathrm{~m}$ distant from the outer surface of a column of radius $0.4 \mathrm{~m}$, after 20 years of exposure to chloride environment. The ramp-type surface concentration model (14) was employed. Spatial steps considered range from $10 \mu \mathrm{m}$ to $0.1 \mathrm{~m}$. For both temporal steps, that is, $\tau=1$ day and 10 days, the chloride concentration remains convergent for $h \leq 5 \mathrm{~mm}$. For larger spatial steps, the chloride concentration diverges and eventually becomes unbounded.

The ideal overlapping between the two curves (Figure 1) seems to suggest that the solution is nearly insensitive to temporal steps. This conjecture is confirmed by the concentration variation as a function of temporal step (Figure 2). For a reasonably small spatial step $(h=1 \mathrm{~mm})$, the chloride concentration becomes slightly diverged when $\tau>10$ days. In view of these arguments, steps $h=1 \mathrm{~mm}$ and $\tau=1$ day were used throughout the subsequent numerical experiments.

3.1. Chloride Diffusion in Unsealed Circular Concretes. As commented by Yang et al. [23], previous studies were primarily concerned with chloride diffusion in one-dimensional concrete. Nonetheless, the size of all real-world concrete structures is finite. A large class of concrete structures, such as viaduct piers, is circular. To investigate the effect of column radius, we monitored the time taken to reach the CTL $\left(1.2 \mathrm{~kg} / \mathrm{m}^{3}\right)$ at the perimeter that is $80 \mathrm{~mm}$ distant from the outer surface of a series of columns. Such a depth $(80 \mathrm{~mm})$ is the minimum value of concrete covers for structures serving under marine environment [34]. For comparison 


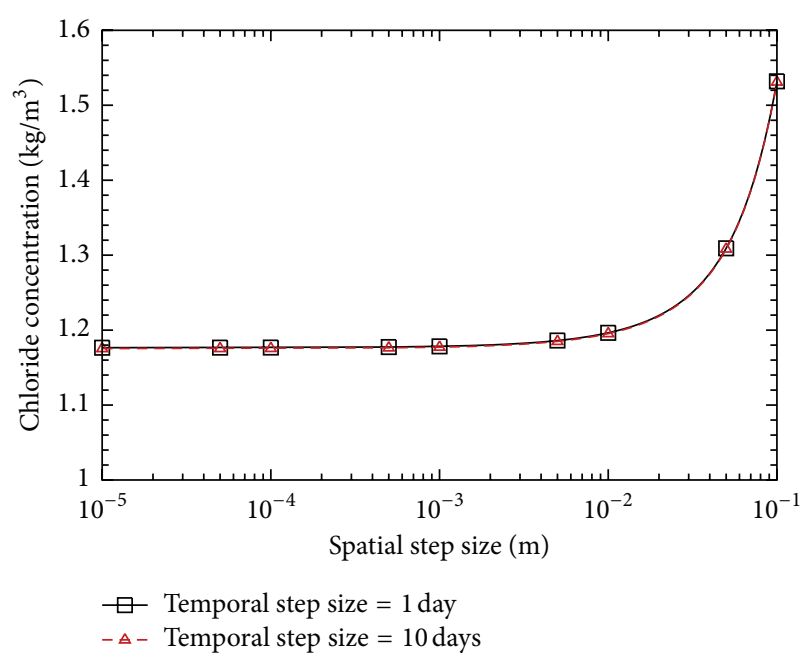

FIGURE 1: The sensitivity of chloride concentration with respect to spatial step $(h)$ for two temporal steps ( $\tau=1$ and 10 days). Concentrations were sampled at $r=0.3 \mathrm{~m}$ of a concrete column of radius $0.4 \mathrm{~m}$, after 20 years of exposure to chloride environments $[6] . w / c=0.5$.

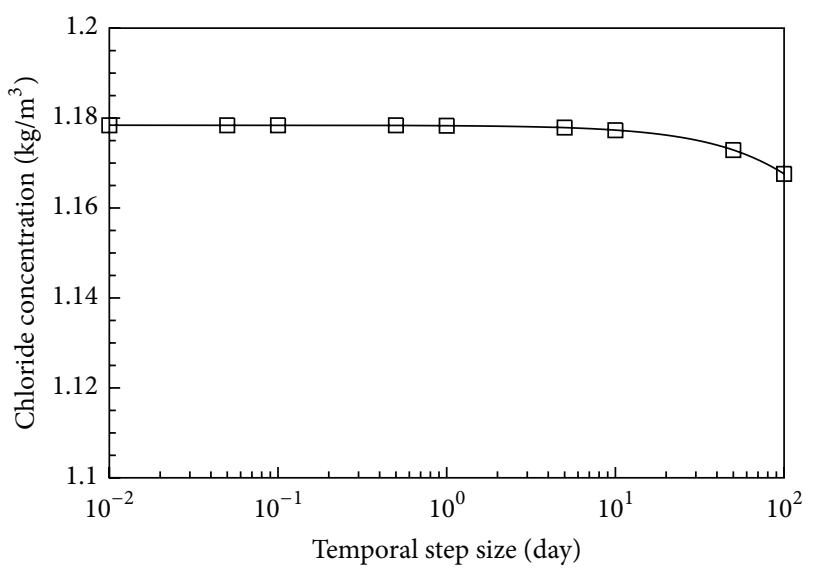

FIGURE 2: The sensitivity of chloride concentration with respect to the temporal step $(\tau)$ for $h=1 \mathrm{~mm}$. Concentrations were sampled under the exact same condition as that of Figure 1.

purpose, years taken to reach the CTL were evaluated for two surface concentration models and three water to cement ratios (Figure 3). For all 6 combinations, the length scale effect is significant for small column sizes and gradually levels off. If the column size were large enough, the column durability converges to its one-dimensional counterpart.

The effect of column radius shows strong dependence on the water to cement ratio and the surface concentration model. As reflected by (17), the water to cement ratio strongly affects the reference diffusivity $D_{w / c}$ (Figure 2 of Song et al. [3]). For the same category of surface concentration model, the smaller the water to cement ratio is, the more years taken to reach the CTL at the concrete cover depth are. The appreciable gaps among the three curves for each surface concentration model suggest the great significance of concrete quality on chloride diffusion. The concrete durability

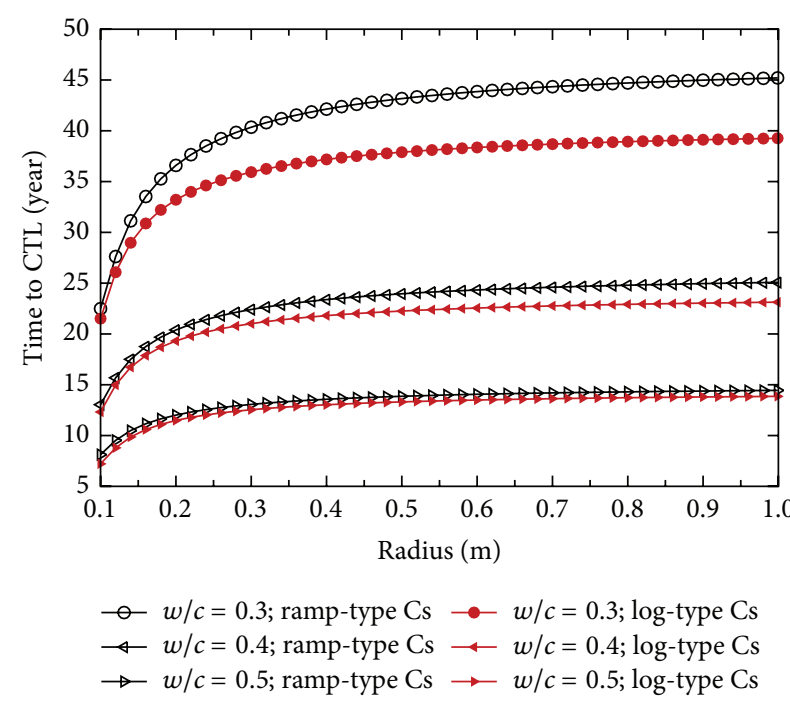

FIGURE 3: Exposure years taken to reach the CTL $\left(1.2 \mathrm{~kg} / \mathrm{m}^{3}\right)$ as a function of column radius. The CTL values were monitored at the minimum concrete depth $(80 \mathrm{~mm})$ [34]. Numerical experiments were performed with respect to three water to cement ratios and two surface concentration models. $R=0.4 \mathrm{~m}$.

(without taking the residual life of a reinforced concrete structure into account) is roughly doubled for each decrease of the three water to cement ratios; that is, $w / c=0.5 \rightarrow$ $0.4 \rightarrow 0.3$.

The durability predictions due to different surface concentration models, that is, (14) and (15), are functions of column radius and water to cement ratio too. For each water to cement ratio, the discrepancy between two curves increases as a function of column size and reaches the maximum as the curvature of radius approaches infinity. In other words, small columns are less sensitive to surface concentration models. This observation can be explained by the smaller area lying between the outer surface and the concrete cover depth $(80 \mathrm{~mm})$. Given the same column size, the discrepancy due to different surface concentration models behaves as a decreasing function of the water to cement ratio. The durability predictions deviate the most for the highest concrete quality $w / c=0.3$. In their one-dimensional solution, Song et al. [3] predicted a service life slightly less than 40 years using $w / c=0.3$ and the log-type surface chloride model (15). With the ramp-type surface concentration model, this service life can be prolonged for more than 5 years (Figure 3 ). This durability improvement is obviously a consequence of the less severe chloride environment associated with the ramp-type model.

To investigate the spatial distribution and temporal evolution, Figure 4 shows a three-dimensional surface plot of the chloride concentration as a function of both exposure time and the radial coordinate of a concrete column of radius $0.4 \mathrm{~m}$. The intermediate level of water to cement ratio $(w / c=0.4)$ was assumed. Given the ramp-type surface concentration model (14), it takes exactly five years for the surface concentration to linearly evolve to the saturated level. The surface concentration subsequently remains at this level. 


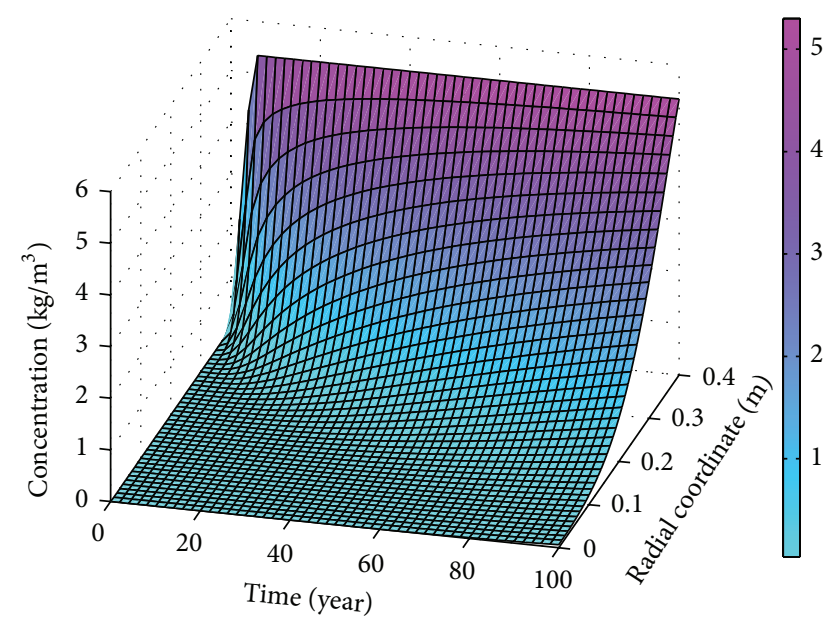

FIGURE 4: The evolution of chloride concentration inside a concrete column of radius $0.4 \mathrm{~m}$. The chloride concentration at the outer surface increases linearly for the first five years of exposure and subsequently remains at the saturated level of $5.343 \mathrm{~kg} / \mathrm{m}^{3}[6] . w / c=$ 0.4 .

Although the chloride concentration monotonically increases with exposure time at an interior radial coordinate $r<R$, the whole evolutionary process can be divided into two distinct stages, in accordance with the two temporal scales of the surface concentration model (14). The concentration evolution curve experiences an inflection point at $t=5$ years and changes from being concave upwards to concave downwards. Should the exposure time be long enough, chloride concentration at any radial coordinate will converge to the saturated surface concentration $\left(C_{0}=5.343 \mathrm{~kg} / \mathrm{m}^{3}\right)$.

To better illustrate the spatial distribution of chloride concentrations, Figure 5 shows 13 concentration profiles at different exposure levels. These curves were all extracted from the surface concentration plot (Figure 4). It is seen that for all exposure scales the chloride concentration is a strong function of the radial coordinate $r$. Chloride anions are primarily confined to the area that is close to the outer surface of a circular column for the beginning years and gradually diffuse toward the column center due to the drive of concentration gradient. Due to the implementation of Neumann boundary condition (10), the concentration gradient is always zero at $r=0$.

For convenience, the CTL $\left(1.2 \mathrm{~kg} / \mathrm{m}^{3}\right)$ for corrosion initiation is also plotted in Figure 5. The coordinates of the points of intersection between the CTL and concentration profiles represent the years taken to reach the CTL at various concrete depths. For the minimum concrete cover depth $80 \mathrm{~mm}$ [34], less than 24 years is required to reach the CTL. For lower graded concrete materials, for example, for $w / c=0.5$, the time to reach the CTL at the minimum concrete depth can be even lower. Such a short time scale greatly threatens the normal functioning of concrete structures and thus proper maintenance strategies must be enforced.

3.2. Chloride Diffusion after Sealer Application. One ideal means to prevent chloride anions from further penetrating

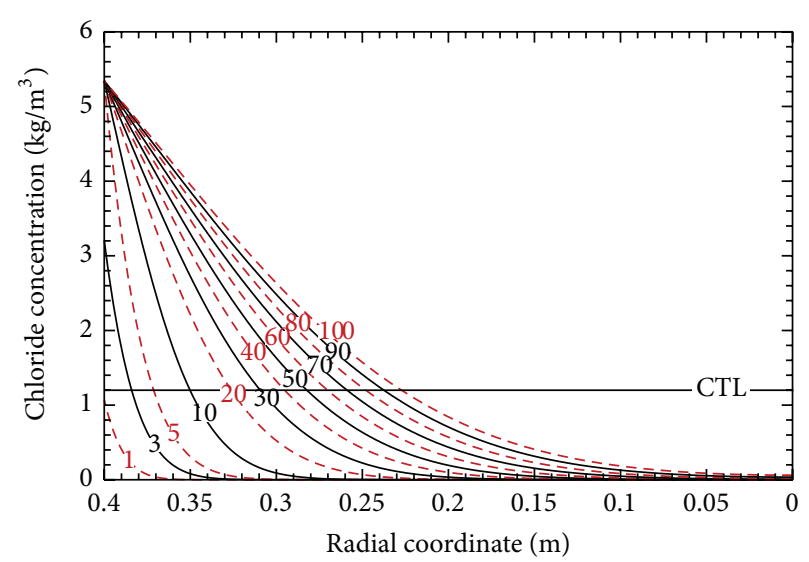

Figure 5: The spatial distribution of chloride concentration for 13 exposure scales, extracted from Figure 4 . Also plotted in the figure is the CTL $\left(1.2 \mathrm{~kg} / \mathrm{m}^{3}\right)$ required for corrosion initiation.

in concrete structures is to remove the concrete covering the top reinforcing layer, to protect the core structure with a sealing membrane, and to restore the concrete cover with fresh concrete. Once a core concrete structure is sealed, no mass transport of chloride anions occurs across the sealer. The diffusion process inside the core structure only involves the redistribution of previously entrapped chloride concentration. A steady state (even distribution over the entire sealed volume) can eventually be expected. The effectiveness of a sealer application is determined by both when and where the strategy is performed. In the worst scenario, the chloride concentration may reach the CTL at the reinforced elements even at the steady state. The corrosion is thus only subjected to the availability of oxygen and moisture in the vicinity of the reinforcements.

In most cases, a sealer application helps reduce the corrosion susceptibility of reinforcements by prohibiting new external chloride ingress and by redistributing entrapped chloride accumulations. Figures 6 and 7 highlight the concentration profiles for a sealer application that is applied at the outer surface and at the minimum concrete cover depth $(80 \mathrm{~mm})$, respectively. For both cases, the sealer was applied after 40 years of exposure to chloride environment. The sealer has a nominal thickness of $5 \mathrm{~mm}$ and is presumably both chloride-free and chloride-proof.

Immediately following the application of a sealer, the passage of external chloride anions was shut down. Mathematically the closure is represented by a Neumann boundary condition. A careful examination on the surface grids of Figure 6 reveals that the chloride concentration for the area between the sealer and approximately the minimum cover depth $(r=0.32 \mathrm{~mm})$ continuously decreases over the simulation period. The chloride concentration in $r<0.32 \mathrm{~m}$, on the other hand, continues to increase in spite of the sealer application. This effect is obviously due to the high chloride concentrations near the sealer. As can be read from Figure 5, the CTL depth prior to the sealer application ( $t=40$ years) is already $0.104 \mathrm{~m}$.

Similar to Figure 5, Figure 7(a) reports the spatial distribution of chloride concentration for 10 exposure scales 


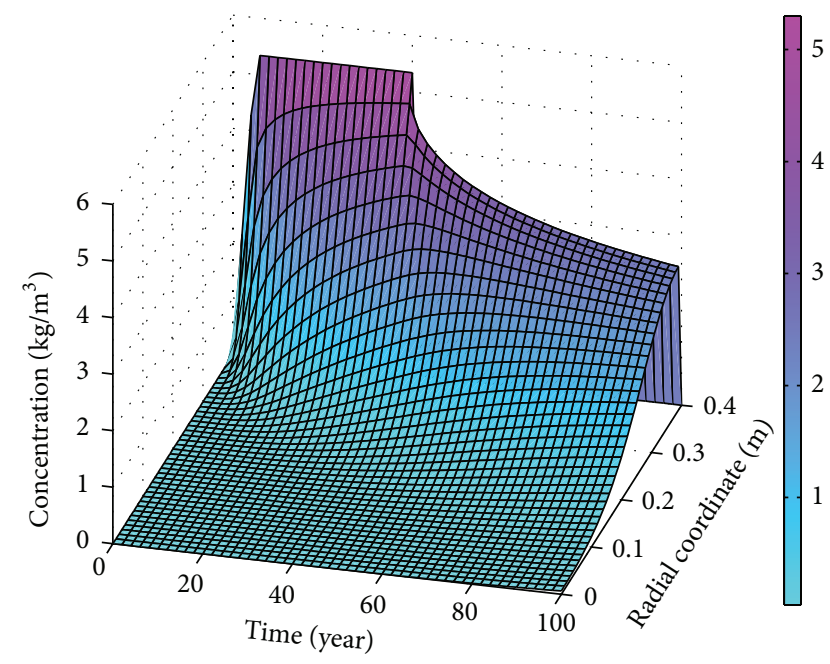

FIGURE 6: The evolution of chloride concentration with a sealing membrane applied at the outer surface of a concrete column, after 40 years. The surface chloride evolution and diffusivity assume the same conditions as those of Figure 4. A nominal thickness of $5 \mathrm{~mm}$ was reserved for the sealing membrane. $R=0.4 \mathrm{~m} . w / c=0.4$.

after a sealer was applied at the outer surface (Figure 6). The crossovers among the 10 curves around $r=0.32 \mathrm{~m}$ confirm the above argument that the chloride concentration in the core region still increases with exposure time. When, in terms of the CTL depth, a direct comparison between Figures 5 and 7(a) suggests that the sealer application at the outer surface results in only marginal improvement on concrete durability. As can be seen from Figure 8, the CTL depths due to an unsealed and sealed column differ from each other only after 25 years after the sealer application. Even after 60 years of the seal application, the relative improvement in CTL depth is less than $9 \%(0.157 \mathrm{~m}$ versus $0.172 \mathrm{~m}$ ). The chloride concentration at the minimum cover depth $(r=0.32 \mathrm{~m})$, however, shows a better improvement (Figure 8). This improvement, unfortunately, does not help much on reducing the CTL depth as reflected in Figures 5 and $7(a)$. The reason can be attributed to the large amount of entrapped chloride content and Neumann boundary conditions enforced at both ends of the radial interval.

To further investigate the impact of sealer application location, Figure 9 reports the chloride profile due to a sealer that was applied at the minimum concrete cover depth $(r=$ $0.32 \mathrm{~m}$ ). The rest of simulation conditions remain the same as those of Figure 6. Due to the fact that most of the chloride accumulations are confined to the near-surface region, the chloride concentration inside the sealer rapidly evolves below the CTL and stays at a low level. The redistribution of chloride concentration in the core concrete follows a similar pattern as that of Figure 6 . The overall magnitude of chloride concentrations, however, is much lower than those of a sealer applied at the outer surface. The sealer application location strongly affects the amount of chloride anions that will be entrapped inside the sealer. These anions are the source of the subsequent redistribution.

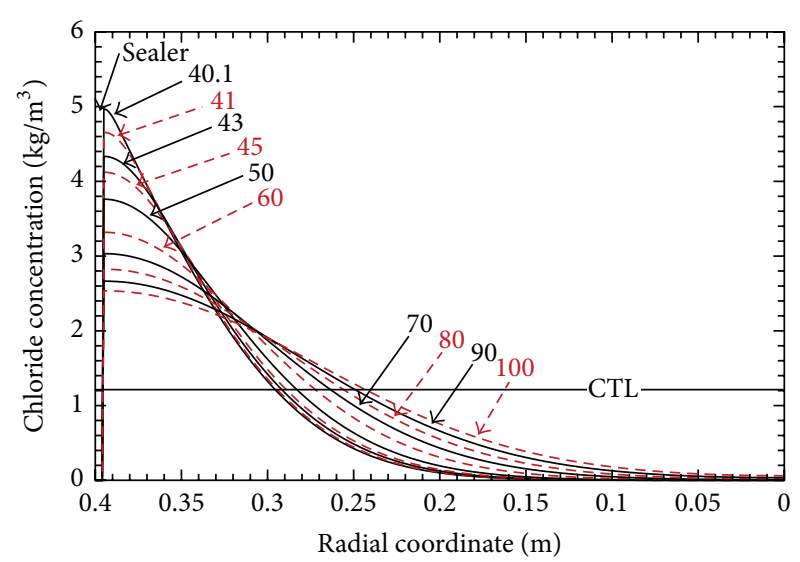

(a)

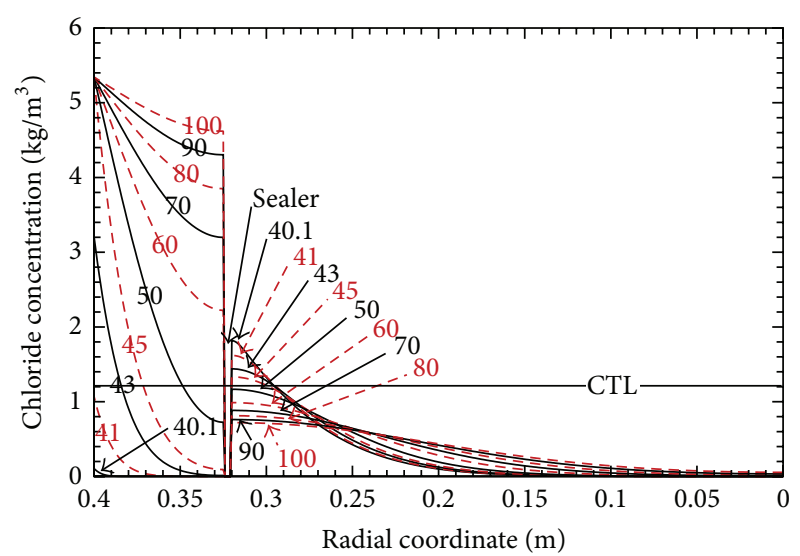

(b)

FIGURE 7: The spatial distribution of chloride concentration for 10 exposure scales, extracted from (a) Figure 6 and (b) Figure 9. Also plotted in each of the two figures is the CTL $\left(1.2 \mathrm{~kg} / \mathrm{m}^{3}\right)$ required for corrosion initiation.

As one cost to apply the sealer at a certain concrete depth, the concrete cover replacement was severely susceptible to chloride penetration. This is due to the shutdown of the penetration passage toward the interior of the concrete column. As a result, chloride anions accumulate at a much faster rate in the concrete cover replacement than in the unsealed concrete columns. Figure 7 (b) shows the spatial distribution of chloride concentrations in both the concrete cover replacement and the core structure for 10 temporal scales after the sealer application. Neumann boundary conditions were enforced at both ends of the sealer. It is important to note that, in less than 10 years after the sealer application, the chloride concentration anywhere in the sealed region evolves below the CTL.

Recall that the sealer application in Figure 9 was performed after 40 years of exposure to chloride environment. For a sealer applied at the minimum cover depth, less than 10 years was required to redistribute the chloride concentration below the CTL (Figure 10). Should a sealer application be applied earlier, even less recovering years are required. In the most ideal scenario, a sealer application should be performed 


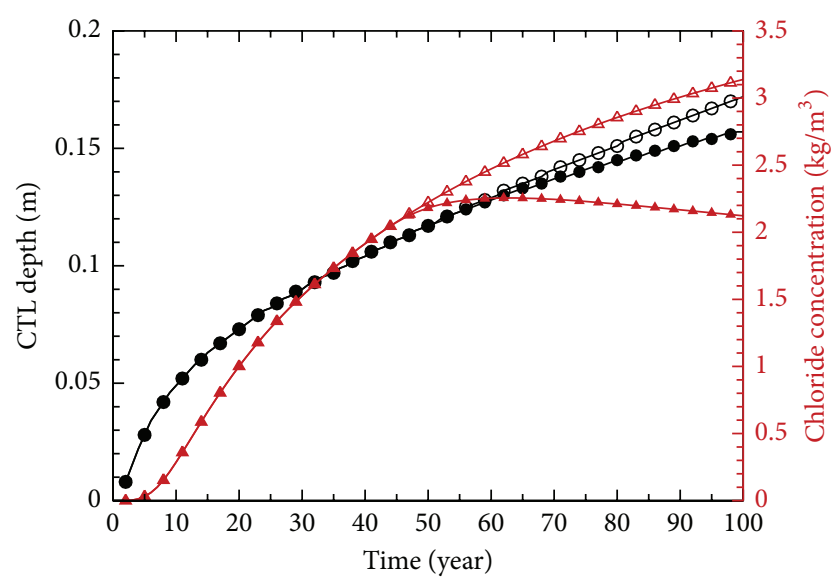

- CTL depth, sealed $\_C(r=320 \mathrm{~mm})$, sealed

- CTL depth, unsealed $\triangle C(r=320 \mathrm{~mm})$, unsealed

FIGURE 8: The evolution of the CTL depth (left vertical axis) and the chloride concentration at the minimum cover depth $(80 \mathrm{~mm})$ (right vertical axis). For each quantity, two scenarios, that is, unsealed and a sealer applied at the outer surface when $t=40$ years, are plotted. $R=0.4 \mathrm{~m} . w / c=0.4$.

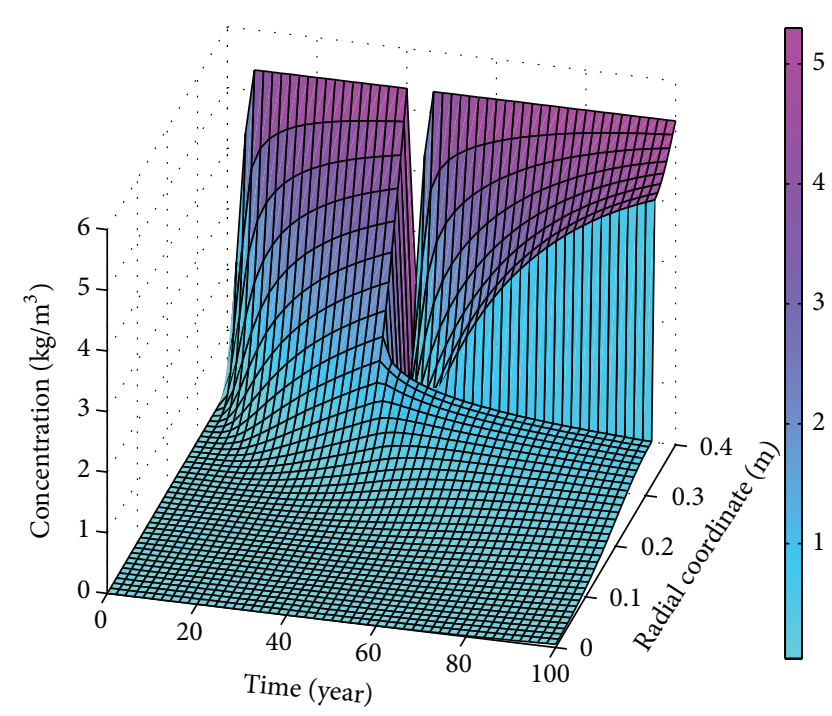

FIGURE 9: The evolution of chloride concentration under the same simulation condition as that of Figure 6, except that the sealer was applied at the minimum cover depth $(80 \mathrm{~mm})$. The sealer application is instantaneously followed by a concrete cover replacement.

when the CTL depth is right about to reach the sealing location (23 and $1 / 2$ years). Sealers applied later than this limit cost more and more years to recover. As can be seen from Figure 10, the functional relationship is nonlinear. For lower quality concrete, for example, $w / c=0.5$, the situation becomes even worse. If the same recovering time is needed, a sealer application must be performed at least 10 years earlier (Figure 10).

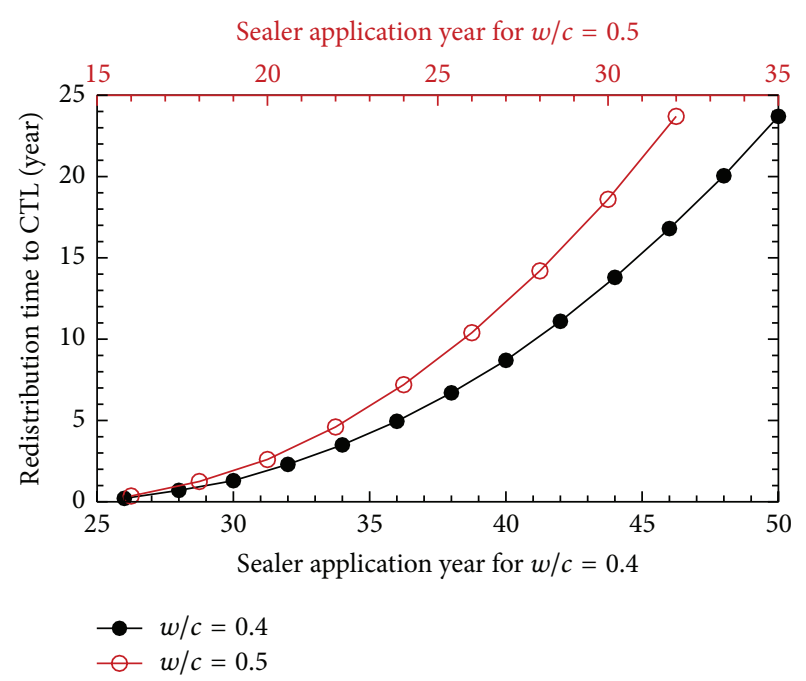

FIGURE 10: Time taken to redistribute chloride concentrations inside a sealer to be less than or equal to the CTL $\left(1.2 \mathrm{~kg} / \mathrm{m}^{3}\right)$. For all samples, the sealer application was applied at the minimum cover depth $(80 \mathrm{~mm}) \cdot R=0.4 \mathrm{~m}$.

\section{Summary and Conclusions}

We analyzed the axially symmetric diffusion problem in a circular domain by developing and implementing a CrankNicolson based finite difference scheme. Particularly, sealer applications were explored as an ideal and somewhat naive protection means for preventing chloride anions from further penetrating in a salt-polluted concrete structure. A few conclusions can be drawn:

(i) In the framework of Fick's laws, chloride diffusion in a (two-dimensional) circular domain is a deterministic process that is governed by the surface concentration model, diffusivity, and initial chloride distribution.

(ii) The significance of size dependency is an inverse function of column radius. For concrete columns large enough $(r \geq 1 \mathrm{~m})$, the present solution for a circular domain, as expected, converges to its onedimensional counterpart.

(iii) An ideal sealer prevents corrosive chlorides from penetrating toward the reinforcements of a concrete structure. Inside the sealer, the amount of chlorides that are available for redistribution is a strong function of the sealer application timing and depth.

(iv) In all cases, a sealer application is advised to be performed early and as close to the outermost reinforcements as possible.

Despite the completeness of this work in the context of an ideal sealer application, future work should attempt to address the following: (1) the incorporation of practical sealers, membranes, and coatings; (2) chloride diffusions across a practical sealer; and (3) the influence of reinforcements on chloride diffusion. 


\section{Appendices}

\section{A. Numerical Solution for an Unsealed Circular Domain}

For an unsealed circular concrete, the proposed finite difference scheme can eventually be reduced to the following system of linear equations:

$$
\begin{gathered}
{\left[\begin{array}{ccccc}
b_{1} & c_{1} & & & \\
a_{2} & b_{2} & c_{2} & & \\
& & \ddots & & \\
& a_{M-1} & b_{M-1} & c_{M-1} \\
& & a_{M} & b_{M}
\end{array}\right]\left\{\begin{array}{c}
C_{1, j} \\
C_{2, j} \\
\vdots \\
C_{M-1, j} \\
C_{M, j}
\end{array}\right\}} \\
=\left\{\begin{array}{c}
f_{1} \\
f_{2} \\
\vdots \\
f_{M-1} \\
f_{M}
\end{array}\right\},
\end{gathered}
$$

where

$$
\begin{aligned}
b_{1}= & \frac{1}{2}+\frac{\tau}{h^{2}} D_{j-1 / 2}, \\
c_{1}= & -\frac{\tau}{h^{2}} D_{j-1 / 2} ; \\
a_{i}= & -\frac{\tau}{2 h^{2}} D_{j-1 / 2}, \\
b_{i}= & 1+\frac{\tau}{h^{2}}\left(1+\frac{1}{2(i-1)}\right) D_{j-1 / 2}, \\
c_{i}= & -\frac{\tau}{2 h^{2}}\left(1+\frac{1}{i-1}\right) D_{j-1 / 2}, \quad i=2, \ldots, M ; \\
f_{1}= & \left(\frac{1}{2}-\frac{\tau}{h^{2}} D_{j-1 / 2}\right) C_{1, j-1}+\frac{\tau}{h^{2}} D_{j-1 / 2} C_{2, j-1}, \\
f_{i}= & -a_{i} C_{i-1, j-1}+\left(2-b_{i}\right) C_{i, j-1}-c_{i} C_{i+1, j-1}, \\
f_{M}= & -a_{M} C_{M-1, j-1}+\left(2-b_{M}\right) C_{M, j-1}-c_{M} C_{M+1, j-1} \\
& -c_{M} C_{M+1, j} .
\end{aligned}
$$

The solution procedure to a system of linear equations should be standard in a textbook focusing on numerical analysis. Since only three diagonal lines of the matrix are nonzero in the present case, we decomposed this matrix into the product of a lower and an upper triangular matrix. For each of them, only two diagonal lines are nontrivia. The solution can therefore be derived by simple forward and backward substitutions.

\section{B. Numerical Solution for a Circular Domain with Sealer Application}

We may apply Neumann boundary condition at an arbitrary internal node $p$ whose spatial coordinate is $r=(p-1) h$. If this node is the outer boundary of a spatial interval, that is, $0 \leq r \leq\left(p_{1}-1\right) h$, the number of equations reduces from $M$ in (A.1) to $p_{1}$. In the new system of linear equations, the first $\left(p_{1}-1\right)$ equations are identical to those of (A.1). However, the three parameters involving the last equation must be revised to reflect Neumann boundary condition:

$$
\begin{aligned}
a_{p 1} & =-\frac{\tau}{h^{2}} D_{j-1 / 2}, \\
b_{p 1} & =1+\frac{\tau}{h^{2}} D_{j-1 / 2}, \\
f_{p 1} & =\frac{\tau}{h^{2}} D_{j-1 / 2} C_{p 1-1, j-1}+\left(1-\frac{\tau}{h^{2}} D_{j-1 / 2}\right) C_{p 1, j-1} .
\end{aligned}
$$

On the other hand, if the node lies on the inner boundary of a spatial interval, that is, $\left(p_{2}-1\right) h \leq r \leq(M-1) h$, only the parameters participating in the first equation of the new system need to be modified:

$$
\begin{aligned}
b_{p 2} & =1+\frac{\tau}{h^{2}} D_{j-1 / 2}, \\
c_{p 2} & =-\frac{\tau}{h^{2}} D_{j-1 / 2}, \\
f_{p 2} & =\left(1-\frac{\tau}{h^{2}} D_{j-1 / 2}\right) C_{p 2, j-1}+\frac{\tau}{h^{2}} D_{j-1 / 2} C_{p 2+1, j-1} .
\end{aligned}
$$

The remaining $\left(M-p_{2}\right)$ equations are identical to the last $\left(M-p_{2}\right)$ equations of (A.1). These two new systems of linear equations can be independently resolved by using the same solution strategy as that of (A.1).

\section{Conflict of Interests}

The authors declare that there is no conflict of interests regarding the publication of this paper.

\section{Acknowledgments}

This work was supported by the Natural Science Foundation of Jiangsu Province (Grant no. BK20130597), the National Natural Science Foundation of China (Grant nos. 11472079 and 11202051), and the Project Sponsored by the Scientific Research Foundation for the Returned Overseas Chinese Scholars, State Education Ministry.

\section{References}

[1] E. Bardal, Corrosion and Protection, Springer, London, UK, 2004.

[2] C. Mi, N. Lakhera, D. A. Kouris, and D. A. Buttry, "Repassivation behaviour of stressed aluminium electrodes in aqueous chloride solutions," Corrosion Science, vol. 54, no. 1, pp. 10-16, 2012. 
[3] H.-W. Song, H.-B. Shim, A. Petcherdchoo, and S.-K. Park, "Service life prediction of repaired concrete structures under chloride environment using finite difference method," Cement and Concrete Composites, vol. 31, no. 2, pp. 120-127, 2009.

[4] K. Y. Ann and H.-W. Song, "Chloride threshold level for corrosion of steel in concrete," Corrosion Science, vol. 49, no. 11, pp. 4113-4133, 2007.

[5] M. K. Kassir and M. Ghosn, "Chloride-induced corrosion of reinforced concrete bridge decks," Cement and Concrete Research, vol. 32, no. 1, pp. 139-143, 2002.

[6] P. Phurkhao and M. K. Kassir, "Note on chloride-induced corrosion of reinforced concrete bridge decks," Journal of Engineering Mechanics, vol. 131, no. 1, pp. 97-99, 2005.

[7] A. Moriwake, Study on durability and maintenance of reinforced con-crete jetty deck against chloride induced deterioration [Ph.D. thesis], Tokyo Institute of Technology, Tokyo, Japan, 1996.

[8] C. Alonso, M. Castellote, and C. Andrade, "Chloride threshold dependence of pitting potential of reinforcements," Electrochimica Acta, vol. 47, no. 21, pp. 3469-3481, 2002.

[9] K. Hong and R. D. Hooton, "Effects of cyclic chloride exposure on penetration of concrete cover," Cement and Concrete Research, vol. 29, no. 9, pp. 1379-1386, 1999.

[10] L. Basheer, J. Kropp, and D. J. Cleland, "Assessment of the durability of concrete from its permeation properties: a review," Construction and Building Materials, vol. 15, no. 2-3, pp. 93-103, 2001.

[11] B. Martín-Pérez, H. Zibara, R. D. Hooton, and M. D. A. Thomas, "Study of the effect of chloride binding on service life predictions," Cement and Concrete Research, vol. 30, no. 8, pp. 1215-1223, 2000.

[12] N. S. Martys, "Diffusion in partially-saturated porous materials," Materials and Structures, vol. 32, no. 222, pp. 555-562, 1999.

[13] G. Lin, Y. H. Liu, and Z. H. Xiang, "Numerical modeling for predicting service life of reinforced concrete structures exposed to chloride environments," Cement and Concrete Composites, vol. 32 , no. 8, pp. 571-579, 2010.

[14] S. H. Han, "Influence of diffusion coefficient on chloride ion penetration of concrete structure," Construction and Building Materials, vol. 21, no. 2, pp. 370-378, 2007.

[15] J. Crank, The Mathematics of Diffusion, Clarendon Press, Oxford, UK, 2nd edition, 1975.

[16] A. V. Saetta, R. V. Scotta, and R. V. Vitaliani, "Analysis of chloride diffusion into partially saturated concrete," ACI Materials Journal, vol. 90, no. 5, pp. 441-451, 1993.

[17] S. Dal Pont, B. A. Schrefler, and A. Ehrlacher, "Experimental and finite element analysis of a hollow cylinder submitted to high temperatures," Materials and Structures, vol. 38, no. 281, pp. 681690, 2005.

[18] M. Alqam, "Transient chloride ion diffusion in a homogeneous concrete column," Arabian Journal for Science and Engineering, vol. 39, no. 5, pp. 3633-3640, 2014.

[19] Y. Wang, L. Y. Li, and C. L. Page, "A two-dimensional model of electrochemical chloride removal from concrete," Computational Materials Science, vol. 20, no. 2, pp. 196-212, 2001.

[20] L. Guo, X. M. Guo, and C. W. Mi, "Multi-scale finite element analysis of chloride diffusion in concrete incorporating paste/aggregate ITZs," Science China: Physics, Mechanics and Astronomy, vol. 55, no. 9, pp. 1696-1702, 2012.

[21] M. Bitaraf and S. Mohammadi, "Analysis of chloride diffusion in concrete structures for prediction of initiation time of corrosion using a new meshless approach," Construction and Building Materials, vol. 22, no. 4, pp. 546-556, 2008.
[22] D. Vořechovská, J. Podroužek, M. Chromá, P. Rovnaníková, and B. Teplý, "Modeling of chloride concentration effect on reinforcement corrosion," Computer-Aided Civil and Infrastructure Engineering, vol. 24, no. 6, pp. 446-458, 2009.

[23] L. F. Yang, Z. Chen, Q. Gao, and J. W. Ju, "Compensation length of two-dimensional chloride diffusion in concrete using a boundary element model," Acta Mechanica, vol. 224, no. 1, pp. 123-137, 2013.

[24] A. K. Suryavanshi, R. N. Swamy, and G. E. Cardew, "Estimation of diffusion coefficients for chloride ion penetration into structural concrete," ACI Materials Journal, vol. 99, no. 5, pp. 441-449, 2002.

[25] L. F. Yang and R. Li, "Analytical study of two-dimensional diffusion of chloride ion in concrete," Advances in Science and Technology of Water Resources, vol. 29, no. 3, pp. 20-23, 2009.

[26] D. P. Bentz, X. P. Feng, and R. D. Hooton, “Time-dependent diffusivities: possible misinterpreation due to spatial dependence," in Proceedings of the 2nd International RILEM Workshop on Testing and Modelling the Chloride Ingress into Concrete, pp. 225-233, Bagneux, France, 2000.

[27] R. E. Weyers, W. P. Chamberlin, and P. C. Hoffman, Concrete Bridge Protection and Rehabilitation: Chemical and Physical Techniques: Service Life Estimates (SHRP-S-668), Strategic Highway Research Program, National Research Council, Washington, DC, USA, 1994.

[28] E. C. Bentz and M. D. A. Thomas, The User Manual of Life-365 Service Life Prediction Model for Reinforced Concrete Exposed to Chlorides, Life-365 Consortium II, Released with Computer Program, 2012.

[29] H.-W. Song, C.-H. Lee, and K. Y. Ann, "Factors influencing chloride transport in concrete structures exposed to marine environments," Cement and Concrete Composites, vol. 30, no. 2, pp. 113-121, 2008.

[30] K. Y. Ann, J. H. Ahn, and J. S. Ryou, "The importance of chloride content at the concrete surface in assessing the time to corrosion of steel in concrete structures," Construction and Building Materials, vol. 23, no. 1, pp. 239-245, 2009.

[31] H.-W. Song, S.-W. Pack, and J.-S. Moon, "Durability evaluation of concrete structures exposed to marine environment focusing on a chloride build-up on concrete surface," in Proceedings of the International Workshop on Life Cycle Management of Coastal Concrete Structures, Nagaoka, Japan, 2006.

[32] A. Iserles, A First Course in the Numerical Analysis of Differential Equations, Cambridge Texts in Applied Mathematics, Cambridge University Press, Cambridge, UK, 1996.

[33] D. U. V. Rosenberg, Methods for the Numerical Solution of Partial Differential Equations, Elsevier, New York, NY, USA, 1969.

[34] A. Costa and J. Appleton, "Chloride penetration into concrete in marine environment-part II: prediction of long term chloride penetration," Materials and Structures, vol. 32, no. 219, pp. 354359, 1999. 

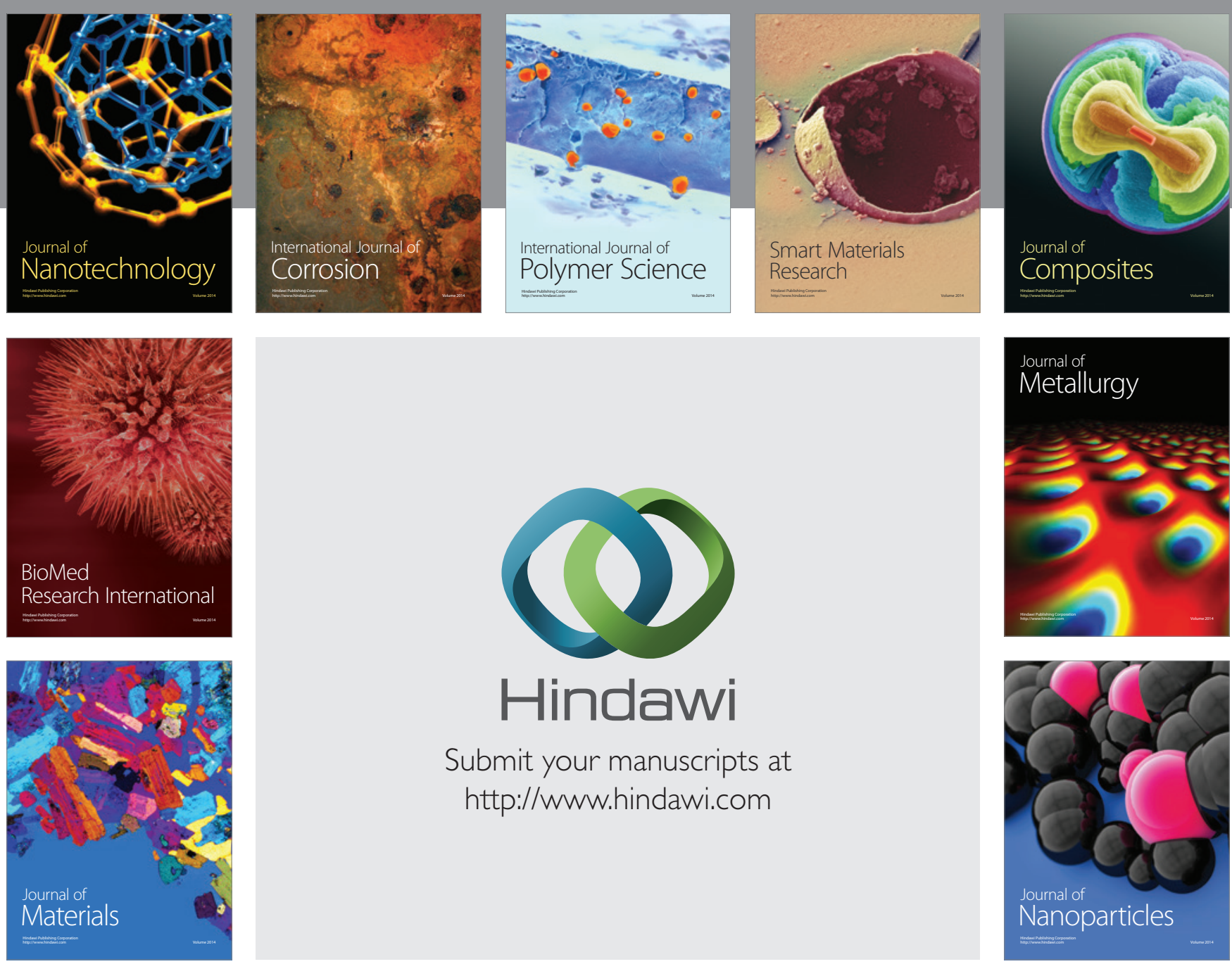

Submit your manuscripts at http://www.hindawi.com
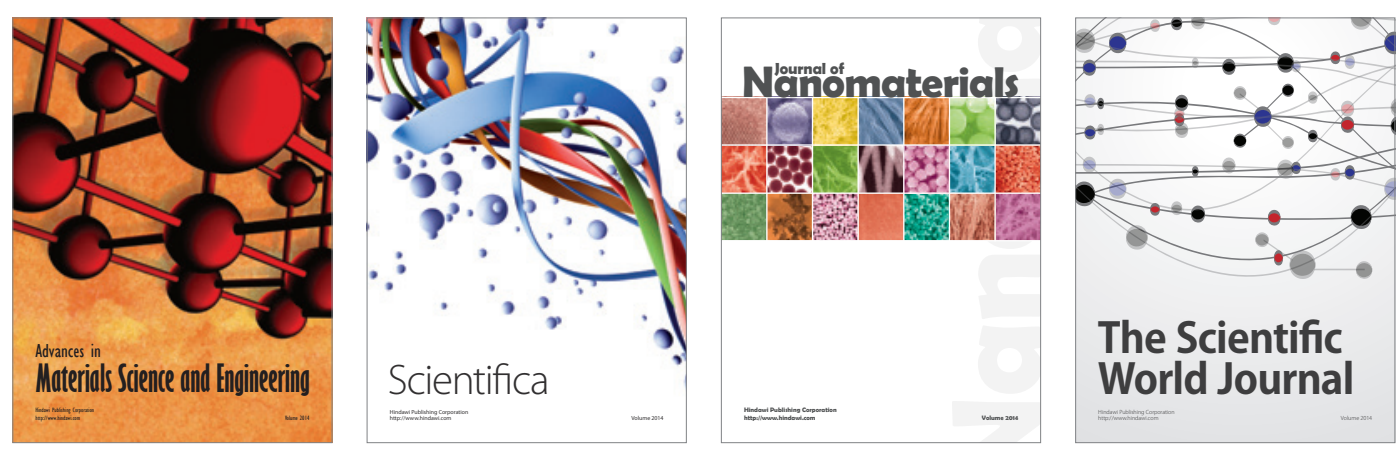

\section{The Scientific World Journal}
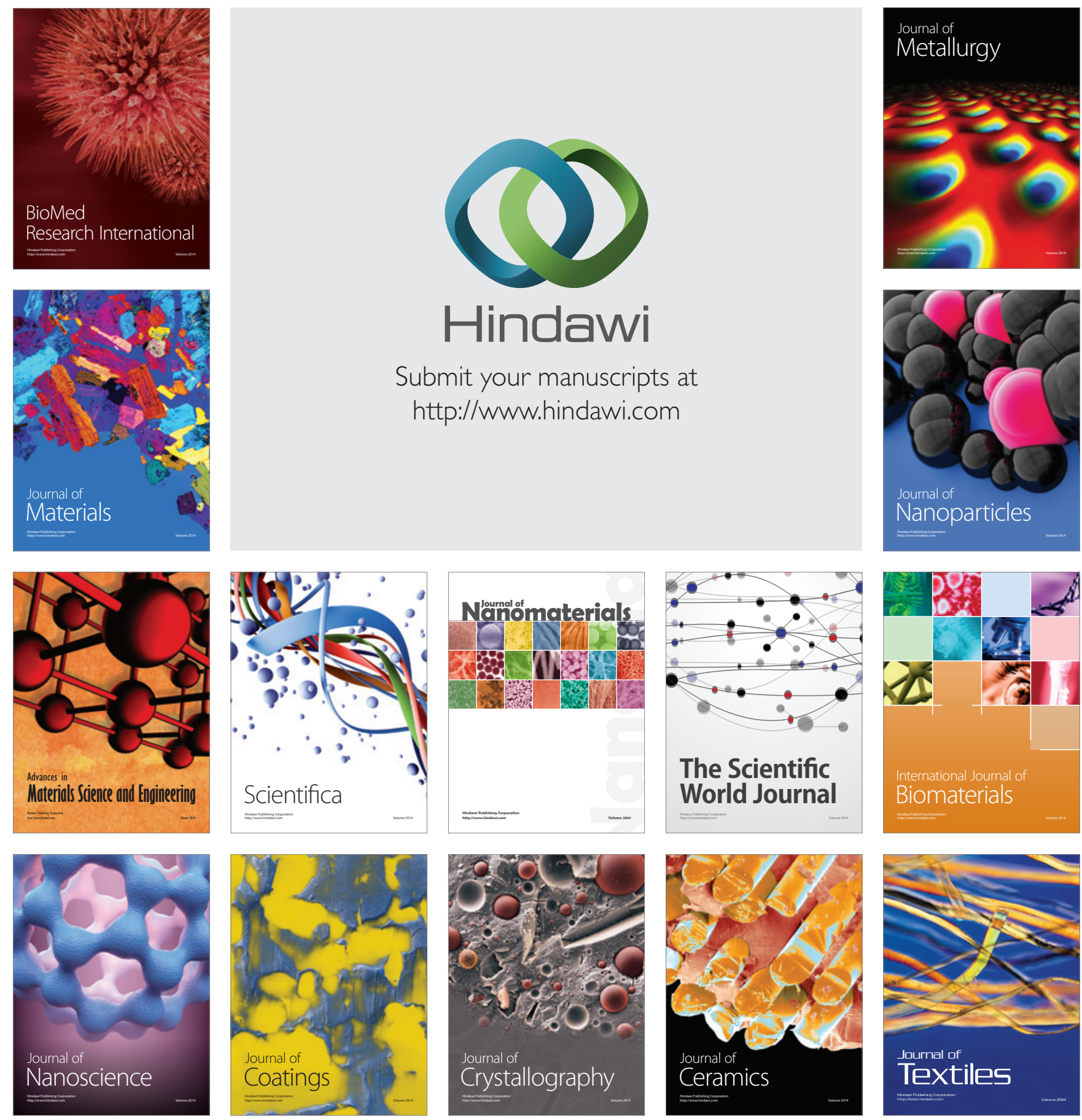